\title{
Enhanced Tyrosine Nitration of Prostacyclin Synthase Is Associated with Increased Inflammation in Atherosclerotic Carotid Arteries from Type 2 Diabetic Patients
}

Chaoyong He, Hyoung Chul Choi, and Zhonglin Xie

From the Department of Medicine, Division of Endocrinology and Diabetes, University of Oklahoma Health Sciences Center, Oklahoma City, Oklaboma

Prostacyclin synthase (PGIS) is tyrosine nitrated in diseased animals. Whether PGIS nitration occurs in human diabetic atherosclerotic arteries has not been reported. The present study was designed to determine PGIS nitration and its association with the inflammatory response in atherosclerotic carotid arteries from patients with or without type 2 diabetes, and carotid plaques were obtained from patients who underwent carotid endarterectomy. PGIS nitration, nitric oxide synthases, adhesion molecules, myeloperoxidase, osteopontin, and matrix metalloproteinase (MMP) were measured by using immunohistochemistry and Western blotting. In low stenosis areas, diabetes enhanced reactive nitrogen species production, as evidenced by increases in 3-nitrotyrosine and PGIS nitration. In parallel, diabetes dramatically increased inflammatory markers including intracellular adhesion molecule-1, vascular adhesion molecule-1, and osteopontin. In both diabetic and nondiabetic patients, MMP-2 and MMP-9 protein levels were significantly increased in the arteries with high stenosis as compared with those with low stenosis. Moreover, diabetes enhanced inducible nitric oxide synthase expression in the plaques from low stenosis areas and up-regulated myeloperoxidase expression in the plaques from both high and low stenosis areas. These data demonstrate that diabetes preferentially increases PGIS nitration that is associated with excessive vascular inflammation in atherosclerotic carotid arteries from patients with type 2 diabetes, suggesting a possible role of tyrosine nitration of PGIS in the development of atherosclerosis in pa- tients with diabetes. (Am J Pathol 2010, 176:2542-2549; DOI: 10.2353/ajpath.2010.090783)

Diabetes mellitus magnifies the risk of macrovascular and microvascular complications. Patients with type 2 diabetes, in particular, have a twofold to fourfold increase in coronary artery disease and peripheral arterial disease. ${ }^{1}$ This has been attributed to several risk factors, including chronic hyperglycemia, dyslipidemia, and insulin resistance, which induce oxidative stress and activate several downstream signals that mediate diabetic complications and render arteries susceptible to atherosclerosis. ${ }^{2}$

Atherosclerosis, formerly considered a lipid storage disease, is now thought to involve an ongoing inflammatory response, which participates in all stages of the disease, from initiation through progression and, ultimately, to the thrombotic complications of atherosclerosis. Accumulating evidence demonstrates that the major mechanisms underlying diabetic vascular complications, such as oxidative stress and endothelial dysfunction, can accelerate atherosclerosis. ${ }^{2}$ These findings provide important links between diabetes and atherosclerosis.

A variety of substances including oxidants, cytokines, and growth factors are involved in the inflammatory process. $^{3}$ One group of these mediators is prostanoids that are produced from arachidonic acid by the action of cyclooxygenase. Among prostanoids, prostacyclin $\left(\mathrm{PGI}_{2}\right)$ and thromboxane A2 ( $\left.\mathrm{TX}_{2}\right)$ have attracted particular attention for their importance in cardiovascular diseases. ${ }^{4}$ The capacity of blood vessels to generate $\mathrm{PGI}_{2}$ is essential to

Supported by an NIH grant (1P20RR024215-01 to Z.X.), a Scientist Development grant from the American Heart Association (Z.X.), and a grant from the Oklahoma Center for Advancement of Science and Technology (Z.X.).

Accepted for publication January 7, 2010.

Address reprint requests to Zhonglin Xie, M.D., Ph.D., Room 302, Basic Science Education Building, Division of Endocrinology and Diabetes, Department of Medicine, University of Oklahoma Health Science Center, 941 Stanton L. Young Blvd, Oklahoma City, OK 73104. E-mail: zxie@ouhsc.edu. 
the integrity of the endothelium. $\mathrm{PGI}_{2}$ is produced in endothelial cells from prostaglandin endoperoxide $\left(\mathrm{PGH}_{2}\right)$ by the action of the enzyme prostacyclin synthase (PGIS). ${ }^{5}$ Inactivation of PGIS causes an accumulation of $P G H_{2}$ and $T X A_{2}$, which activate thromboxane $A_{2}$ receptors (TP), inducing a striking inflammatory response in vasculature. ${ }^{6,7}$

Oxidative stress is a key mediator in the development and progression of diabetic vascular complications. ${ }^{8}$ Many basic and clinical studies demonstrate that hyperglycemia-enhanced oxidative stress induces endothelial dysfunction, an early marker of diabetic complications. In vitro, exposure of isolated arteries to a high concentration of glucose attenuates endothelium-dependent relaxation. ${ }^{9}$ Consistently, in vivo studies have also shown that hyperglycemia induces endothelial dysfunction in diabetic and nondiabetic subjects. ${ }^{10,11}$ In addition, the acute effects of hyperglycemia are counterbalanced by antioxidants. ${ }^{12-14}$ Superoxide over production, when accompanied by increased nitric oxide generation, favors the formation of a highly reactive species, peroxynitrite $\left(\mathrm{ONOO}^{-}\right) \cdot{ }^{15-17} \mathrm{ONOO}^{-}$can initiate both nitrosative and oxidative reactions with proteins, lipids, and DNA. A characteristic reaction of $\mathrm{ONOO}^{-}$is the nitration of proteinbound tyrosine residues. ${ }^{15-17} \mathrm{ONOO}^{-}$has been reported to induce endothelial dysfunction through a mechanism that depends on the nitration of PGIS. ${ }^{18}$ For example, exogenous $\mathrm{ONOO}^{-}$inactivates PGIS by nitration modification, leading to reduction of $\mathrm{PGI}_{2}$ and accumulation of vasoconstrictor prostanoids ${ }^{18}$ in vasculature harvested from diabetic animals. ${ }^{19-21}$

Although PGIS is found to be nitrated in diseased animals, ${ }^{22}$ whether PGIS nitration occurs in human diabetic arteries as well whether it associates with the development of atherosclerosis in patients with diabetes have not been reported. We, therefore, evaluated the relationship between the presence of PGIS nitration and inflammation in human carotid arteries with low- and high-degrees of stenosis from patients with or without type 2 diabetes.

\section{Materials and Methods}

All investigations were approved by the institutional review board of the hospital, and informed consent was obtained from all patients. The study group comprised 21 type 2 diabetic patients and 19 nondiabetic patients with asymptomatic carotid stenosis. The patients were enlisted to undergo carotid endarterectomy for extracranial high-grade $(>70 \%)$ internal carotid artery stenosis. ${ }^{23}$ The degree of luminal narrowing was determined by repeated Doppler echography and intra-arterial cerebral angiography using the North American Symptomatic standard criteria. Diabetes was categorized according to the American Diabetes Association criteria. ${ }^{24}$ Patients with diabetes were taking metformin as an antidiabetic therapy. The clinical characteristics of the patients are summarized in Table 1.

\section{Atherectomy Specimens}

After surgery, the specimens were divided into two parts: one part was from a low stenosis area (edge area of the
Table 1. Characteristics of Study Patients

\begin{tabular}{lcc}
\hline \multicolumn{1}{c}{ Variables } & Nondiabetes & Diabetes \\
\hline$N$ & 19 & 21 \\
Age, y & $66 \pm 4$ & $69 \pm 5$ \\
Sex, male/female & $10 / 9$ & $11 / 10$ \\
Hypertension & 19 & 21 \\
Fasting blood glucose, mg/dl & $90.5 \pm 11.0$ & $130.2 \pm 42^{*}$ \\
HbA1c, \% & $5.4 \pm 0.1$ & $7.8 \pm 0.9^{*}$ \\
Triglyceride, mg/dl & $128.7 \pm 63.4$ & $149.3 \pm 76.1$ \\
Active therapy, $n$ & & \\
Statin & 10 & 15 \\
Insulin & - & - \\
Metformin & - & 21 \\
ACE-inhibitor & 8 & 8 \\
CCB & 8 & 6 \\
\hline
\end{tabular}

Data expressed as mean $\pm \mathrm{SE}$ unless noted otherwise. ACEinhibitor, angiotensin II converting enzyme inhibitor; CCB, calcium channel blocker; -, no patient was treated by this medicine.

${ }^{*} P<0.01$ versus nondiabetes.

plaque; Figure $1 \mathrm{~A}$ ) and another from a high stenosis area (central area of the plaque; Figure 1B). The specimen was immediately immersion fixed in $10 \%$ buffered formalin. Sections were serially cut in $5 \mu \mathrm{m}$, mounted on lysinecoated slides, and stained by the H\&E method. Carotid artery specimens were analyzed by light microscopy.

\section{Immunohistochemistry}

Sections were deparaffinized, rehydrated, and blocked with $5 \%$ normal serum. The sections were then incubated with specific antibodies: antiendothelial nitric oxide synthase (eNOS; BD Transduction Laboratories, San Jose, CA), anti-inducible nitric oxide synthase (iNOS; BD Transduction Laboratories), anti-myeloperoxidase (MPO; Santa Cruz Biotechnology, Inc., Santa Cruz, CA), anti-intercellular adhesion molecule-1 (ICAM-1; Santa Cruz Biotechnology, Inc.), anti-vascular cell adhesion molecule 1 (VCAM-1; Santa Cruz Biotechnology, Inc.), anti-osteopontin (Abcam, Cambridge, MA), antimatrix metalloproteinase (MMP)-2 (Calbiochem, San Diego, CA), and MMP-9 (Novocastra Laboratories Ltd., Benton Lane, UK). The sections were then incubated for 30 minutes with secondary antibodies; 3, 3'-Diaminobenzidine or Avidin fluorescent D (Vector Laboratories, Burlingame, CA) was used to visualize positive immunoreactivity. Normal rabbit or mouse immunoglobulin fractions were substituted to primary antibodies as negative control. Double staining for 3-nitrotyrosine (Upstate Biotechnology, Inc., Billerica, MA) and PGIS (Cayman Chemical, Ann Arbor, MI) was performed by using a kit from Vector Laboratories, followed the protocol provided by the company, and was viewed on a SLM 510 laser scanning confocal microscope (Carl Zeiss Meditec, Inc., Jena, Germany).

\section{Quantitative Analysis for Histology}

Analysis of experiments was performed with a personalcomputer-based 8-bit color image-analysis system. In brief, the sections were visualized under an Olympus inverted microscope (Olympus America, Melville, NY). 

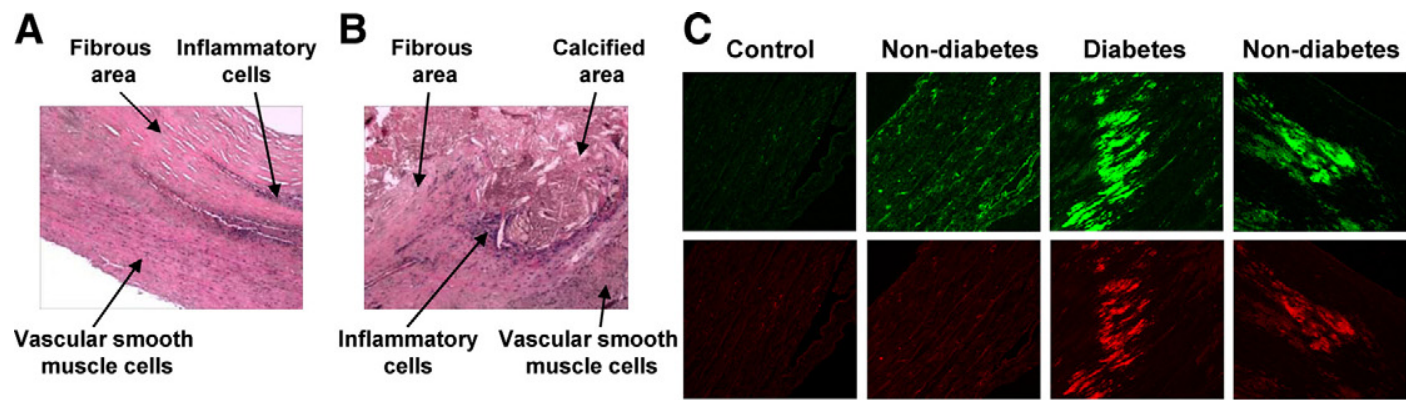

Diabetes
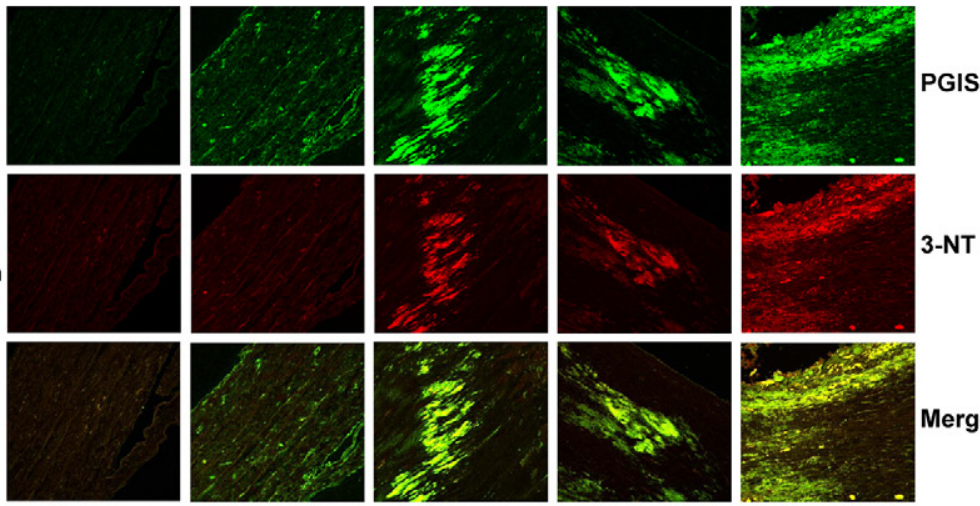

Figure 1. Colocalization of 3-nitrotyrosine (3-NT) and PGIS in atherosclerotic plaques from diabetic and nondiabetic patients. A and B: Representative example from low stenosis stained with H\&E. C: Representative examples of double staining of atherosclerotic plaques. Top: immunostaining for PGIS (green) in atherosclerotic plaques. Middle: immunoreactivity of 3-NT (red) from the same section. Bottom: merged images from 3-NT and PGIS staining; note that 3-NT staining (red) is colocalized with PGIS staining (green), and yellow color indicates the colocalization of 3-NT immunoreactivity and PGIS staining. Normal rabbit or mouse immunoglobulin fractions were substituted to primary antibodies as a negative control. $\mathbf{D}$ and $\mathbf{E}$ : Quantitative analyses of immunohistochemical staining of 3-NT and PGIS are shown. All of the data represent the mean $\pm \mathrm{SEM}$; nondiabetic group, $n=$ 19; diabetic group, $n=21$.

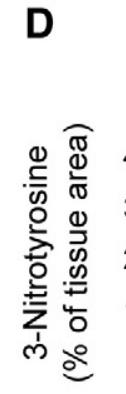

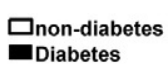

Low Stenosis

Diabetes
E

High Stenosis

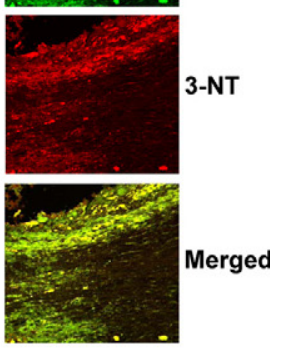

口non-diabetes



Sequence images were taken by using a Retiga 1300 color-cooled camera and Q capture software (QImaging, Burnaby, BC, Canada). The staining areas were measured by using a Bioquant analysis system (Nashville, TN), and a color threshold mask for immunostaining was defined to detect the red color by sampling. The same threshold was applied to all specimens. The percentage of the total area with positive color for each section was recorded.

\section{Cell Culture and Treatments}

Human aortic endothelial cells (HAECs; American Type Culture Collection, Rockville, MD) were cultured in Ham's F12 medium, 10\% fetal bovine serum, $100 \mu \mathrm{g} / \mathrm{ml}$ heparin, and $30 \mu \mathrm{g} / \mathrm{ml}$ endothelial cell growth supplements. Cells were seeded in $60 \times 15 \mathrm{~mm}$ culture dishes precoated with $0.1 \%$ pig gelatin. When they reached confluence, the cells were used for the experiments. HAECs were incubated with palmitate $(100 \mu \mathrm{mol} / \mathrm{L})$, high glucose (30 $\mathrm{mmol} / \mathrm{L}$ ), or palmitate plus high glucose for 72 hours. After incubation, cellular proteins were extracted and the cell lysates were subjected to immunoprecipitation and Western blot analysis.

\section{Immunoprecipitation and Western Blot Analysis}

Nitration of PGIS in cell extract was identified by immunoprecipitation and Western blotting as previously described.25-27 Total cell lysates were prepared in lysis buffer (1\% Triton X-100, $150 \mathrm{mmol} / \mathrm{L} \mathrm{NaCl}, 10 \mathrm{mmol} / \mathrm{L}$ Tris, $\mathrm{pH} 7.4,1 \mathrm{mmol} / \mathrm{L}$ EDTA, $1 \mathrm{mmol} / \mathrm{L}$ EGTA, $0.2 \mathrm{mmol} / \mathrm{L}$ phenylmethylsulfonyl fluoride, $0.2 \mathrm{mmol} / \mathrm{L}$ sodium or- thovanadate, and $0.5 \%$ Nonidet P-40), and protein contents were measured by using the Bradford assay. Solubilized proteins were incubated with $10 \mu \mathrm{g}$ of monoclonal antinitrotyrosine antibody. Immune complexes were precipitated with protein A Sepharose CL-4B, resolved by SDS-polyacrylamide gel electrophoresis, and blotted on to a nitrocellulose membrane. The membranes were then incubated with appropriate primary antibodies and analyzed as described previously. ${ }^{28,29}$

\section{Assay of PG/S Activity}

PGIS activity was determined by measuring the stable metabolite of $\mathrm{PGI}_{2}, 6$-keto-PGF ${ }_{1 \alpha}$, after cells were incubated with its substrate, $\mathrm{PGH}_{2}\left(10^{-5} \mathrm{~mol} / \mathrm{L}\right.$ for 3 minutes $)$, as described previously. ${ }^{18,30}$ Briefly, after incubation with $\mathrm{PGH}_{2}$, the reaction was stopped by acidification with $1 \mathrm{~N}$ $\mathrm{HCl}$ to $\mathrm{pH}$ 3.5. Incubation media were extracted with ethyl acetate. After centrifugation, the organic phases were evaporated to dryness under nitrogen. Samples were then resuspended in $100 \mu \mathrm{l}$ of PBS. The amount of 6-keto-PGF ${ }_{1 \alpha}$ and $\mathrm{PGE}_{2}$ was subsequently determined with an enzyme-linked immunosorbent assay kit (Cayman Chemicals) according to the instructions provided by the supplier.

\section{Statistical Analysis}

Data are presented as mean \pm SE. Significance was determined by one-way analysis of variance followed by Bonferroni's method. $P<0.05$ was considered statistically significant. 
A

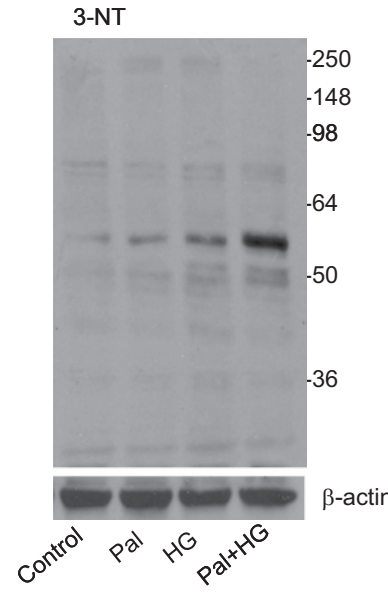

B

$$
\text { IP: PGIS }
$$

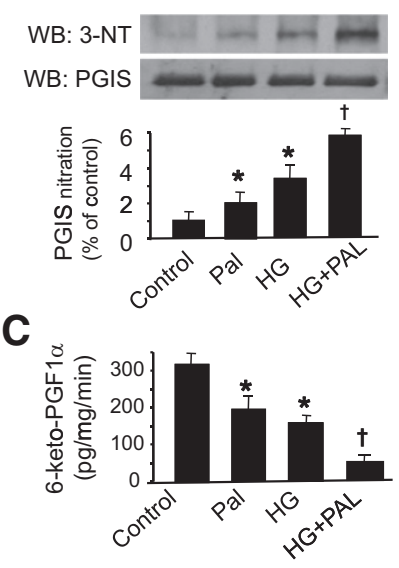

Figure 2. High glucose and palmitate increase prostacyclin synthase nitration and decrease its activity in HAECs. A: HAECs were incubated with palmitate (Pal; $100 \mu \mathrm{mol} / \mathrm{L})$, high glucose $(\mathrm{HG} ; 30 \mathrm{mmol} / \mathrm{L})$, or high glucose plus palmitate for 72 hours. After incubation, cellular proteins were extracted as described in Materials and Methods, and the cell lysates were subjected to Western blot analysis by using an antibody against 3-nitrotyrosine (3-NT) The blot is a representative of three blots from three different experiments. B: PGIS was immunoprecipitated (IP) from the HAECs treated with palmitate high glucose, or high glucose plus palmitate; PGIS and 3-NT were detected in Western blots (WB). The blot is a representative of three blots from three different experiments. C: PGIS activity was assessed by analyzing $\mathrm{PGF}_{\alpha 1}$, a degradation component of $\mathrm{PGI}_{2}$, using enzyme-linked immunoassay, $n=5$. ${ }^{*} P<0.05$ versus control; ${ }^{\dagger} P<0.05$ versus palmitate or high glucose.

\section{Results}

\section{Diabetes Induces Prostacyclin Synthase Nitration in Carotid Plaques}

We set out to investigate whether PGIS is nitrated in carotid plaques by using a double-labeling immunofluorescence technique. We first detected the formation of 3-nitrotyrosine in carotid plaques obtained from diabetic and nondiabetic patients, which is generally regarded as an index of reactive nitrogen species. In the plaques from both diabetic and nondiabetic patients, the staining areas of 3-nitrotyrosine

were significantly increased in high stenosis areas as compared with those in low stenosis areas. Diabetes induced more 3-nitrotyrosine formation in the plaques from both high and low stenosis areas (Figure 1, C and D). Similar to the alterations of 3-nitrotyrosine, the expression of PGIS was increased in plaques from high stenosis areas in both diabetic and nondiabetic patients. In both areas, diabetic plaques had a higher PGIS level (Figure 1, C and E). Importantly, the merged confocal images of green and red fluorescence provided a yellow color to indicate the areas of colocalization of anti-PGIS and anti-nitrotyrosine in the plaques, which demonstrated identical staining patterns with these two antibodies (Figure 1C), indicating that PGIS is a major protein nitrated by $\mathrm{ONOO}^{-}$in the carotid plaques. These findings suggest that diabetes increases reactive nitrogen species production, resulting in tyrosine nitration of PGIS.

\section{Hyperglycemia and Palmitate Increase PGIS Nitration and Reduced Its Activity}

Two hallmarks of type 2 diabetes are hyperglycemia and increased free fatty acids. To further confirm that diabetes induces tyrosine nitration of PGIS, we treated HAECs with high glucose to mimic hyperglycemia and palmitate to mimic elevated free fatty acids for 72 hours. Compared with control group, high glucose and palmitate alone increased 3-nitrotyrosine levels. Of note, a combination of high glucose and palmitate proved to be much more potent in inducing oxidative stress (Figure 2A). As a result, PGIS nitration, analyzed by immunoprecipitation and Western blot, was increased in the cells exposed to high glucose or palmitate alone, and high glucose plus palmitate additively increased nitrated PGIS (Figure 2B). PGIS activity, judged by measuring the decomposition product of $\mathrm{PGI}_{2}$, 6-keto-PGF ${ }_{1 \alpha}$, was dramatically decreased in the cells treated with high glucose or palmitate. Notably, a combination of high glucose and palmitate further decreased PGIS activity (Figure 2C). These in
A



Low Stenosis
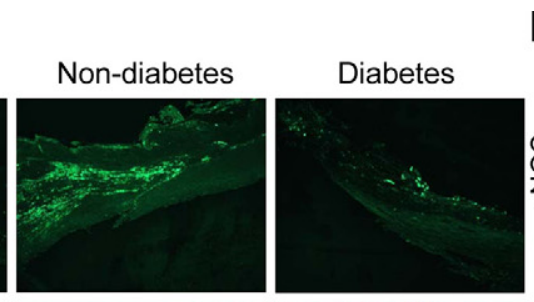

B



High Stenosis



C
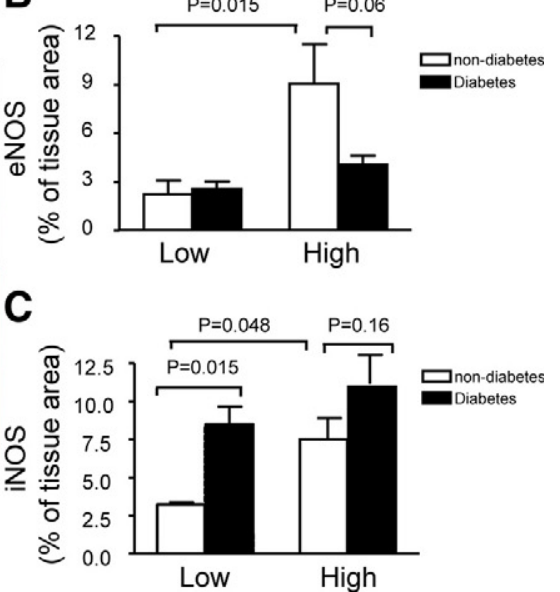

Figure 3. Morphological analysis of eNOS and iNOS expression in carotid plaques from diabetic and nondiabetic patients. A: Representative sections show specific immunoreactivity for eNOS (green signal; top). Bottom: Representative photomicrographs demonstrate iNOS immunoreactivities (red signal). B and C: Quantitative morphometric measurement of immunohistochemistry for eNOS and iNOS is shown. Data are expressed as mean \pm SEM; nondiabetic group, $n=$ 19 ; diabetic group, $n=21$. 


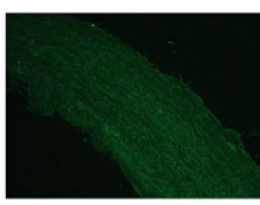

Low Stenosis

B

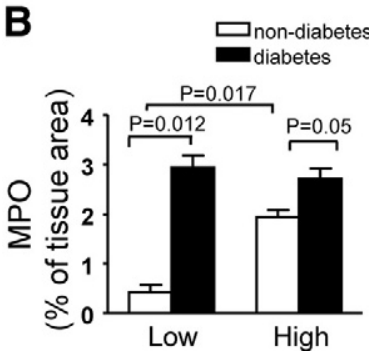



C

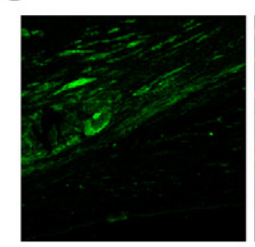

MPO



High Stenosis

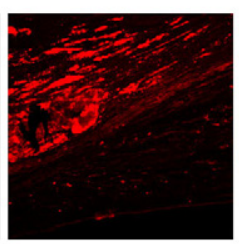

3-NT

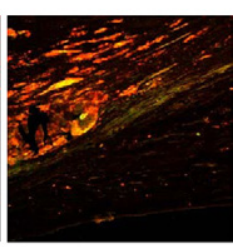

Merged

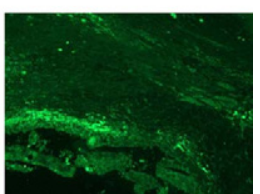

. Diabetes increases MPO expression in carotid plaques. A: Immunohistochemistry demonstrates that MPO is expressed differently in diabetic and nondiabetic carotid plaques. B: Quantification of MPO immunostaining is shown. Image analysis indicates that diabetes increases MPO expression in the plaques from both low and high stenosis areas. Data are expressed as mean \pm SEM; these results are typical of 21 diabetic and 19 nondiabetic plaques. C: 3-NT and MPO double staining in atherosclerotic plaques is shown. vitro experiments support that diabetes increases reactive nitrogen species production, induces PGIS nitration, and inhibits PGIS activity.

\section{Modulation of eNOS, iNOS, and MPO Expression by Diabetes}

Next we examined the potential sources of reactive nitrogen species by staining for eNOS, iNOS, and MPO. In nondiabetic plaques, eNOS expression was elevated in high stenosis areas. Diabetic plaques from high stenosis areas revealed a weak staining of eNOS as compared with nondiabetic plaques (Figure $3, A$ and $B$ ). In the plaques from low stenosis areas, immunoreactivity of iNOS was more abundant in patients with diabetes. In the plaques from nondiabetic patients, the immunoreactivity of iNOS was enhanced in the high stenosis areas compared with the low stenosis areas (Figure 3, A and C). Myeloperoxidase, a leukocyte-derived enzyme, catalyzes the formation of a number of reactive oxidant species and has emerged as a potential participant in the development of atherosclero- sis. Immunohistochemical staining for MPO was enhanced by diabetes in both low and high stenosis areas. In nondiabetic plaques, it was increased in high stenosis areas (Figure 4, A and B). Double staining of 3-nitrotyrosine and MPO showed the staining region of 3-nitrotyrosine was larger than that of MPO, thus there was only partial overlap in these two stainings (Figure 4C).

\section{Alternations of Inflammatory Markers in Diabetic and Nondiabetic Carotid Plaques}

PGIS has been shown to be nitrated and inactivated in early stage atherosclerotic lesions in animal models, ${ }^{22}$ and inhibition of PGIS increases endothelial inflammation via TP receptor stimulation in cultured endothelial cells. ${ }^{6}$ To investigate whether nitrated PGIS correlates with the inflammatory response in human atherosclerotic plaques, we stained several inflammatory markers, including ICAM-1, VCAM-1, and osteopontin in patients with diabetes. Diabetic plaques from low stenosis areas revealed more intensive staining of ICAM-1 than nondia-

A

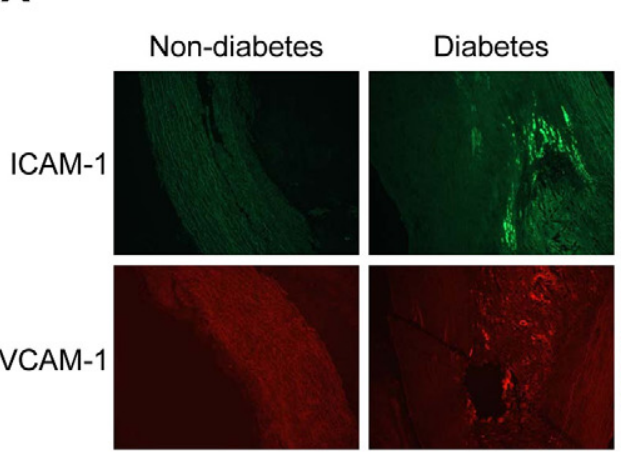

Low Stenosis



High Stenosis
B

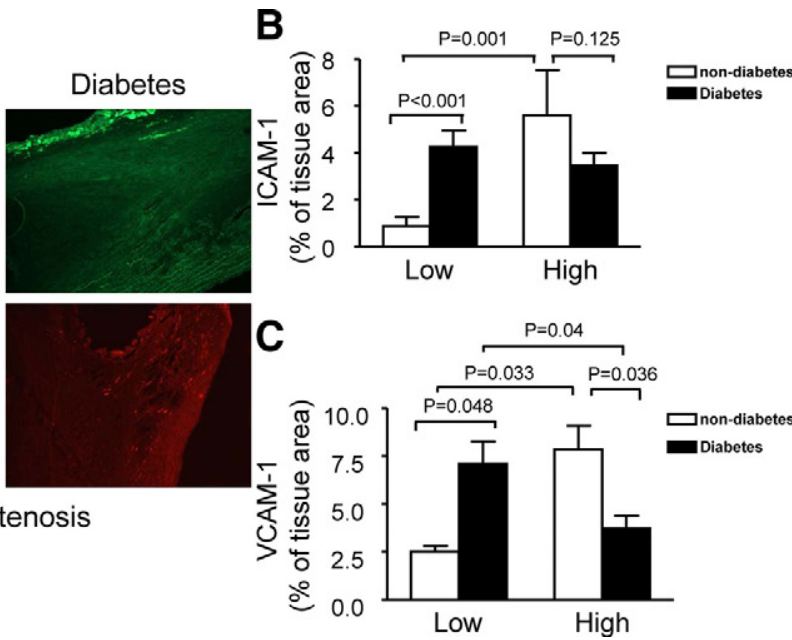

Figure 5. Immunohistochemical detection of ICAM-1 and VCAM- 1 in the plaques from diabetic and nondiabetic patients. A: Top: VCAM- 1 was immunostained with a specific antibody (red signal). Bottom: Representative immunostaining for ICAM-1 (green signal). B and C: Quantification of immunohistochemical staining for VCAM-1 and ICAM-1 is shown. All of the data are expressed as mean \pm SEM; nondiabetic group, $n=19$; diabetic group, $n=21$. 
A

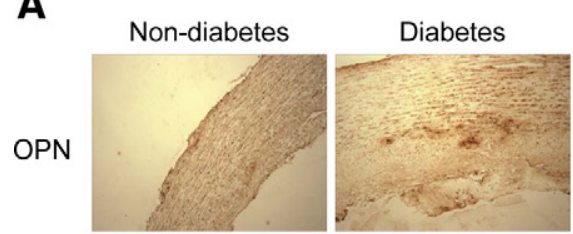

Low Stenosis

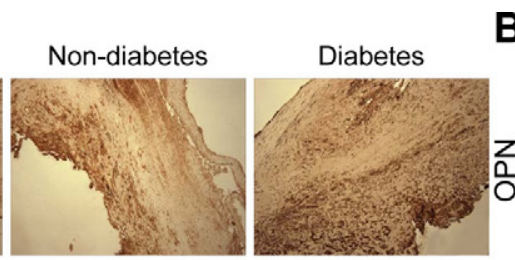

High Stenosis



Figure 6. Expression of osteopontin in human atherosclerotic plaques from diabetic and nondiabetic patients. A: Representative photomicrographs demonstrate the expression of osteopontin (brown signal) on paraffin sections form diabetic and nondiabetic patients. B: Quantitative analysis for OPN immunostaining. Data are expressed as mean \pm SEM from 21 diabetic and 19 nondiabetic patients.

betic plaques. Immunohistochemical staining for ICAM-1 was elevated in the plaques from high stenosis areas in nondiabetic patients (Figure 5, A and B). Similarly, immunostaining of VCAM-1 was more abundant in the diabetic sections from low stenosis areas and the staining of VCAM-1 was increased in high stenosis areas in nondiabetic plaques (Figure 5, A and C). Osteopontin (OPN), initially identified in osteoblasts as a mineralization-modulatory matrix protein, is a multifunctional protein and up-regulated in a variety of acute and chronic inflammatory conditions, including wound healing, fibrosis, and atherosclerosis. OPN is highly expressed in atherosclerotic plaques, especially in macrophages and foam cells. In the context of atherosclerosis, OPN is generally regarded as a proinflammatory and proatherogenic molecule. $^{31}$ In both diabetic and nondiabetic patients, osteopontin staining was enhanced in the plaques from high stenosis areas. Osteopontin expression was elevated in diabetic plaques from low stenosis areas as compared with nondiabetic plaques (Figure 6, A and B). Thus, these analyses confirmed the presence of a higher inflammatory response in diabetic plaques.

\section{Expression of Matrix Metalloproteinase in the Plaques from Diabetic and Nondiabetic Patients}

Matrix metalloproteinase has been consistently implicated in the pathophysiology of atherosclerosis and plaque rupture. To determine the expression of MMP in patients' atherosclerotic plaques, we analyzed the protein levels of MMP-2 and MMP-9 in the carotid plaques from diabetic and nondiabetic patients by using immunohistochemistry with specific antibodies. As shown in Figure 7, MMP-2 staining was significantly increased in the plaques from high stenosis areas in both diabetic and nondiabetic patients (Figure 7, A and B). Similar to the change in 3-nitroyrosine (Figure 1, C and D), diabetes dramatically increased MMP-9 levels in the plaques from both low and high stenosis areas (Figure 7, A and C).

\section{Discussion}

In this investigation, we demonstrate that in human carotid artery plaques from low stenosis areas, where atherosclerosis initiates, diabetes enhanced tyrosine nitration of PGIS, accompanied by significant increases in inflammatory markers including ICAM-1, VCAM-1, and osteopontin. In diabetic plaques from high stenosis areas, increased PGIS nitration was associated with an increase in MMP-9 protein levels. Moreover, diabetes enhanced iNOS expression in the plaques from low stenosis areas and up-regulated MPO expression in both high and low stenosis areas. These findings support that tyrosine nitration of PGIS is correlated with an excessive inflammatory response in atherosclerotic carotid arteries from patients with type 2 diabetes.

Diabetes mellitus increases oxidative and nitrosative stress, triggering the development of endothelial dys-

A

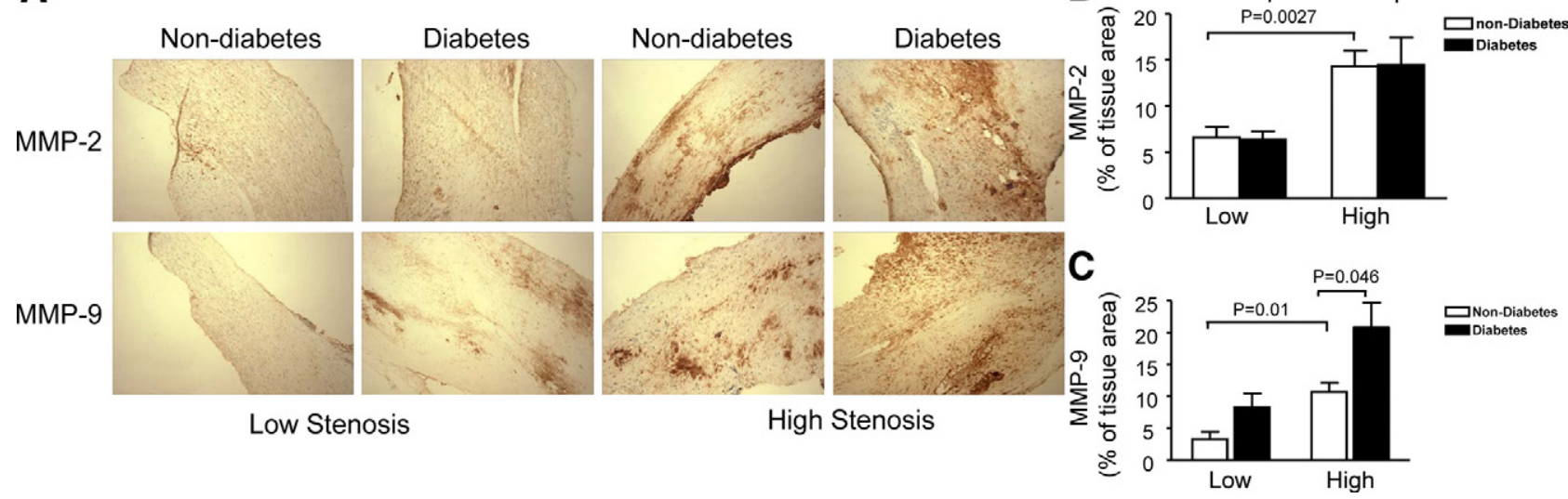

Figure 7. Immunohistochemical analysis for MMP-2 and MMP-9 in carotid plaques. A: Top: Representative cross sections show specific immunoreactivity for MMP-2. Bottom: Immunostaining of carotid atherosclerotic lesions for MMP-9 is shown. Quantification of MMP-2 (B) and MMP-9 (C) immunohistochemical stainings is shown. Data are expressed as mean \pm SEM from 21 diabetic and 19 nondiabetic patients. 
function, an early event of atherosclerosis. ${ }^{32,33}$ Vascular cells are capable of generating $\mathrm{ONOO}^{-}$because of their capacity to simultaneously release superoxide and NO. Previous studies have demonstrated that high glucose enhances superoxide production in vascular cells, ${ }^{6}$ which may arise from NADPH oxidase, xanthine oxidase, the mitochondrial respiratory chain, the arachidonic acid cascade including lipooxygenase and cyclooxygenase, and microsomal enzymes. ${ }^{34}$ The effect of high glucose on eNOS in cultured endothelial cells is a subject of debate because evidence for both an increase ${ }^{33}$ and a decrease ${ }^{35}$ have been published in the literature. In this study we did not find a significant difference in eNOS expression between diabetic and nondiabetic patients in the plaques from the low stenosis areas, although the expression of eNOS seemed to be reduced by diabetes in the plaque from the high stenosis areas. Our data suggest that increased NO may result from elevated expression of iNOS. Cromheeke et $\mathrm{al}^{36}$ have reported colocalization of iNOS and nitrotyrosine in atherosclerotic plaques, and the authors indicated that iNOS was enzymatically active in the plaques that produced NO, and $\mathrm{NO}$ reacted with superoxide to form $\mathrm{ONOO}^{-}$. In turn, $\mathrm{ONOO}^{-}$nitrated tyrosine residues of proteins. ${ }^{37}$

MPO is a mammalian enzyme critical for the innate immune defenses and other inflammatory processes. It is present in granulocytes ${ }^{36}$ and macrophages ${ }^{38}$ in human atherosclerotic plaques; MPO plays a role in catalyzing oxidative and nitrative modification of targeted proteins in the human arterial wall. In the current study we found that diabetes increased MPO expression in both low and high stenosis areas, and there was only a partial overlay in 3-nitrotyrosine and MPO stainings, suggesting that MPO partially contributes to the 3-nitrotyrosine formation in atherosclerotic plaques. These results are consistent with our previous findings that hypocholorous acid, a product of MPO, increases $\mathrm{ONOO}^{-}$and superoxide production via NADPH oxidase, resulting in eNOS uncoupling and endothelial dysfunction. ${ }^{39}$

Although NO is known as an important vasodilator, its role in atherogenesis is controversial. NO may prevent atherogenesis by reducing oxidative stress, monocyte recruitment, and the proliferation of smooth muscle cells. However, high doses of NO promote atherogenesis by inducing apoptosis, matrix breakdown, and $\mathrm{ONOO}^{-}$formation. ${ }^{40}$ In the present study, we provide convincing evidence that diabetes enhances $\mathrm{ONOO}^{-}$formation in carotid arteries in both low and high stenosis areas. We have also shown that the staining areas of 3-nitrotyrosine were significantly larger in the plaques from patients with diabetes, and in vitro high glucose plus palmitate increased 3-nitrotyrosine formation. Importantly, using a double staining technique, we found that 3-nitrotyrosine staining was mainly colocalized with the PGIS staining, suggesting that PGIS might be a major protein nitrated by $\mathrm{ONOO}^{-}$in humans. Nitration of PGIS by $\mathrm{ONOO}^{-}$may be an important mechanism for diabetic vascular complications. Eicosanoaids are important players in the normal homeostasis of the endothelium. TXA 2 is a potent agonist of platelet aggregation, thrombus formation, and vasoconstriction, whereas $\mathrm{PGI}_{2}$ leads to an increase in CAMP that deactivates platelets, inhibits clot formation, and is a potent vasodilator. In endothelial and smooth muscle cells, $\mathrm{ONOO}^{-}$readily nitrates and inactivates PGIS at an extremely low concentration with a reported (half maximal inhibitory concentration) $\mathrm{IC}_{50}=$ $100 \mathrm{nmol} / \mathrm{L} .{ }^{41}$ Inactivation of PGIS results in reduction of $\mathrm{PGI}_{2}$ and accumulation of $\mathrm{PGH}_{2}$ within vascular tissues, which activates TP receptor, ${ }^{6,7}$ triggering platelet aggregation, thrombus, vasospasm, cell apoptosis, and expression of adhesion molecule. ${ }^{6}$ Previous studies show that $\$ 18886$, a TP antagonist, increased eNOS expression and reduced inflammatory markers in the vasculature of diabetic mice. ${ }^{7}$ In agreement with these data, we found that diabetes increased ICAM-1 and VCAM-1 expression in the plaques from the low stenosis areas. Moreover, the expression of osteopontin, a proinflammatory cytokine, was also elevated by diabetes. Thus, nitration of PGIS by $\mathrm{ONOO}^{-}$likely represents an important mechanism for inflammatory response in diabetic arteries, probably contributing to acceleration of atherosclerosis in patients with type 2 diabetes.

In the plaques of high stenosis areas from patients with diabetes, increased PGIS nitration is associated with an increase in MMP-9 protein levels. Matrix metalloproteinases belong to a family of zinc-dependent endopeptidases that promote degradation of components in the extracellular matrix. ${ }^{42}$ Recently, several lines of evidence have demonstrated that MMPs might weaken the fibrous cap and subsequently destabilize atherosclerotic lesion. ${ }^{43}$ Elevated glucose induces MMP-2 and MMP-9 expression and increases their activity. ${ }^{44,45}$ Inhibition of TP receptor by S188886 reduces MMP expression and activity. ${ }^{46}$ Overall these reports suggest stimulation of TP receptor may not only promote the progression of atherogenesis but also transform lesions toward a destabilized phenotype. Therefore, the possibility of a TP inhibitor preventing plaque destabilization deserves further investigation in the future.

In summary, we have reported that hyperglycemia increases oxidative stress and tyrosine nitration of PGIS, resulting in reduction in $\mathrm{PGI}_{2}$ and accumulation of $\mathrm{PGH}_{2}$ and $\mathrm{TXA}_{2}$. Associated activation of TP receptors increases inflammatory response in the vasculature, ${ }^{6,7}$ a major pathological process of atherosclerosis. Thus, diabetes-enhanced tyrosine nitration of PGIS may play a role in acceleration of atherosclerosis in patients with diabetes.

\section{Acknowledgment}

We thank Dr. Ming-Hui Zou for many helpful discussions.

\section{References}

1. Beckman JA, Creager MA, Libby P: Diabetes and atherosclerosis: epidemiology, pathophysiology, and management. JAMA 2002, 287 : 2570-2581

2. Biondi-Zoccai GG, Abbate A, Liuzzo G, Biasucci LM: Atherothrombosis, inflammation, and diabetes. J Am Coll Cardiol 2003, 41:10711077

3. Libby P, Ridker PM, Maseri A: Inflammation and atherosclerosis Circulation 2002, 105:1135-1143

4. Kobayashi T, Tahara $Y$, Matsumoto M, Iguchi M, Sano H, Murayama T, Arai $H$, Oida H, Yurugi-Kobayashi T, Yamashita JK, Katagiri H, 
Majima M, Yokode M, Kita T, Narumiya S: Roles of thromboxane A(2) and prostacyclin in the development of atherosclerosis in apoEdeficient mice. J Clin Invest 2004, 114:784-794

5. Linton MF, Fazio S: Cyclooxygenase-2 and atherosclerosis. Curr Opin Lipidol 2002, 13:497-504

6. Zou MH, Shi C, Cohen RA: High glucose via peroxynitrite causes tyrosine nitration and inactivation of prostacyclin synthase that is associated with thromboxane/prostaglandin $\mathrm{H}(2)$ receptor-mediated apoptosis and adhesion molecule expression in cultured human aortic endothelial cells. Diabetes 2002, 51:198-203

7. Zuccollo A, Shi C, Mastroianni R, Maitland-Toolan KA, Weisbrod RM, Zang M, Xu S, Jiang B, Oliver-Krasinski JM, Cayatte AJ, Corda S, Lavielle G, Verbeuren TJ, Cohen RA: The thromboxane A2 receptor antagonist S18886 prevents enhanced atherogenesis caused by diabetes mellitus. Circulation 2005, 112:3001-3008

8. Ceriello A: New insights on oxidative stress and diabetic complications may lead to a "causal" antioxidant therapy. Diabetes Care 2003, 26:1589-1596

9. Bohlen HG, Lash JM: Topical hyperglycemia rapidly suppresses EDRF-mediated vasodilation of normal rat arterioles. Am J Physiol 1993, 265:H219-H225

10. Giugliano D, Marfella R, Coppola L, Verrazzo G, Acampora R, Giunta R, Nappo F, Lucarelli C, D'Onofrio F: Vascular effects of acute hyperglycemia in humans are reversed by L-arginine: evidence for reduced availability of nitric oxide during hyperglycemia. Circulation 1997, 95:1783-1790

11. Kawano H, Motoyama T, Hirashima O, Hirai N, Miyao Y, Sakamoto T, Kugiyama K, Ogawa H, Yasue H: Hyperglycemia rapidly suppresses flow-mediated endothelium-dependent vasodilation of brachial artery. J Am Coll Cardiol 1999, 34:146-154

12. Tesfamariam B, Cohen RA: Free radicals mediate endothelial cell dysfunction caused by elevated glucose. Am J Physiol 1992, 263 : H321-H326

13. Marfella R, Verrazzo G, Acampora R, La MC, Giunta R, Lucarelli C, Paolisso G, Ceriello A, Giugliano D: Glutathione reverses systemic hemodynamic changes induced by acute hyperglycemia in healthy subjects. Am J Physiol 1995, 268:E1167-E1173

14. Ting HH, Timimi FK, Boles KS, Creager SJ, Ganz P, Creager MA: Vitamin C improves endothelium-dependent vasodilation in patients with non-insulin-dependent diabetes mellitus. J Clin Invest 1996, 97:22-28

15. Beckman JS, Beckman TW, Chen J, Marshall PA, Freeman BA: Apparent hydroxyl radical production by peroxynitrite: implications for endothelial injury from nitric oxide and superoxide. Proc Natl Acad Sci USA 1990, 87:1620-1624

16. Beckman JS, Koppenol WH: Nitric oxide, superoxide, and peroxynitrite: the good, the bad, and ugly. Am J Physiol 1996, 271:C1424-C1437

17. Beckman JS, Chen J, Ischiropoulos H, Crow JP: Oxidative chemistry of peroxynitrite. Methods Enzymol 1994, 233:229-240

18. Zou MH, Ullrich V: Peroxynitrite formed by simultaneous generation of nitric oxide and superoxide selectively inhibits bovine aortic prostacyclin synthase. FEBS Lett 1996, 382:101-104

19. Tesfamariam B, Jakubowski JA, Cohen RA: Contraction of diabetic rabbit aorta caused by endothelium-derived PGH2-TXA2. Am J Physiol 1989, 257:H1327-H1333

20. Dai FX, Diederich A, Skopec J, Diederich D: Diabetes-induced endothelial dysfunction in streptozotocin-treated rats: role of prostaglandin endoperoxides and free radicals. J Am Soc Nephrol 1993, 4:1327-1336

21. Shimizu K, Muramatsu M, Kakegawa Y, Asano H, Toki Y, Miyazaki Y, Okumura K, Hashimoto $\mathrm{H}$, Ito $\mathrm{T}$ : Role of prostaglandin $\mathrm{H} 2$ as an endothelium-derived contracting factor in diabetic state. Diabetes 1993, 42:1246-1252

22. Zou MH, Leist M, Ullrich V: Selective nitration of prostacyclin synthase and defective vasorelaxation in atherosclerotic bovine coronary arteries. Am J Pathol 1999, 154:1359-1365

23. Young B, Moore WS, Robertson JT, Toole JF, Ernst CB, Cohen SN, Broderick JP, Dempsey RJ, Hosking JD: An analysis of perioperative surgical mortality and morbidity in the asymptomatic carotid atherosclerosis study. ACAS Investigators. Asymptomatic Carotid Artheriosclerosis Study. Stroke 1996, 27:2216-2224

24. Report of the expert committee on the diagnosis and classification of diabetes mellitus: Diabetes Care 2003, 26(Suppl 1):S5-S20
25. Xie Z, Dong Y, Scholz R, Neumann D, Zou MH: Phosphorylation of LKB1 at serine 428 by protein kinase $\mathrm{C}$-zeta is required for metforminenhanced activation of the AMP-activated protein kinase in endothelial cells. Circulation 2008, 117:952-962

26. Xie Z, Zhang J, Wu J, Viollet B, Zou MH: Upregulation of mitochondrial uncoupling protein-2 by the AMP-activated protein kinase in endothelial cells attenuates oxidative stress in diabetes. Diabetes 2008, 57:3222-3230

27. Xie Z, Dong Y, Zhang M, Cui MZ, Cohen RA, Riek U, Neumann D, Schlattner U, Zou MH: Activation of protein kinase $\mathrm{C}$ zeta by peroxynitrite regulates LKB1-dependent AMP-activated protein kinase in cultured endothelial cells. J Biol Chem 2006, 281:6366-6375

28. Xie Z, Dong Y, Zhang J, Scholz R, Neumann D, Zou MH: Identification of the serine 307 of LKB1 as a novel phosphorylation site essential for its nucleocytoplasmic transport and endothelial cell angiogenesis. Mol Cell Biol 2009, 29:3582-3596

29. Xie Z, Singh M, Siwik DA, Joyner WL, Singh K: Osteopontin inhibits interleukin-1beta-stimulated increases in matrix metalloproteinase activity in adult rat cardiac fibroblasts: role of protein kinase C-zeta. J Biol Chem 2003, 278:48546-48552

30. Zou MH, Klein T, Pasquet JP, Ullrich V: Interleukin 1beta decreases prostacyclin synthase activity in rat mesangial cells via endogenous peroxynitrite formation. Biochem J 1998, 336(Pt 2):507-512

31. Cho HJ, Cho HJ, Kim HS: Osteopontin: a multifunctional protein at the crossroads of inflammation, atherosclerosis, and vascular calcification. Curr Atheroscler Rep 2009, 11:206-213

32. Calles-Escandon J, Cipolla M: Diabetes and endothelial dysfunction: a clinical perspective. Endocr Rev 2001, 22:36-52

33. Cosentino F, Hishikawa K, Katusic ZS, Luscher TF: High glucose increases nitric oxide synthase expression and superoxide anion generation in human aortic endothelial cells. Circulation 1997, 96:25-28

34. Brownlee M: Biochemistry and molecular cell biology of diabetic complications. Nature 2001, 414:813-820

35. Ding Y, Vaziri ND, Coulson R, Kamanna VS, Roh DD: Effects of simulated hyperglycemia, insulin, and glucagon on endothelial nitric oxide synthase expression. Am J Physiol Endocrinol Metab 2000, 279:E11-E17

36. Cromheeke KM, Kockx MM, De Meyer GR, Bosmans JM, Bult H, Beelaerts WJ, Vrints CJ, Herman AG: Inducible nitric oxide synthase colocalizes with signs of lipid oxidation/peroxidation in human atherosclerotic plaques. Cardiovasc Res 1999, 43:744-754

37. White CR, Brock TA, Chang LY, Crapo J, Briscoe P, Ku D, Bradley WA, Gianturco SH, Gore J, Freeman BA: Superoxide and peroxynitrite in atherosclerosis. Proc Natl Acad Sci USA 1994, 91:1044-1048

38. Daugherty A, Dunn JL, Rateri DL, Heinecke JW: Myeloperoxidase, a catalyst for lipoprotein oxidation, is expressed in human atherosclerotic lesions. J Clin Invest 1994, 94:437-444

39. Xu J, Xie Z, Reece R, Pimental D, Zou MH: Uncoupling of endothelial nitric oxidase synthase by hypochlorous acid: role of $\mathrm{NAD}(\mathrm{P}) \mathrm{H}$ oxidase-derived superoxide and peroxynitrite. Arterioscler Thromb Vasc Biol 2006, 26:2688-2695

40. Bult $\mathrm{H}$ : Nitric oxide and atherosclerosis: possible implications for therapy. Mol Med Today 1996, 2:510-518

41. Zou M, Jendral M, Ullrich V: Prostaglandin endoperoxide-dependent vasospasm in bovine coronary arteries after nitration of prostacyclin synthase. Br J Pharmacol 1999, 126:1283-1292

42. Galis ZS, Khatri JJ: Matrix metalloproteinases in vascular remodeling and atherogenesis: the good, the bad, and the ugly. Circ Res 2002, 90:251-262

43. Galis ZS, Sukhova GK, Lark MW, Libby P: Increased expression of matrix metalloproteinases and matrix degrading activity in vulnerable regions of human atherosclerotic plaques. J Clin Invest 1994, 94: 2493-2503

44. Kadoglou NP, Daskalopoulou SS, Perrea D, Liapis CD: Matrix metalloproteinases and diabetic vascular complications. Angiology 2005 , 56:173-189

45. Death AK, Fisher EJ, McGrath KC, Yue DK: High glucose alters matrix metalloproteinase expression in two key vascular cells: potential impact on atherosclerosis in diabetes. Atherosclerosis 2003, 168:263-269

46. Viles-Gonzalez JF, Fuster V, Corti R, Valdiviezo C, Hutter R, Corda S, Anand SX, Badimon JJ: Atherosclerosis regression and TP receptor inhibition: effect of S18886 on plaque size and composition: a magnetic resonance imaging study. Eur Heart J 2005, 26:1557-1561 